\title{
Schematic and realistic model calculations of the isovector spin monopole excitations in ${ }^{116}$ In
}

\author{
D. R. Bes, ${ }^{1}$ O. Civitarese, ${ }^{2}$ and J. Suhonen ${ }^{3}$ \\ ${ }^{1}$ Department of Physics, Laboratory Tandar, Centro Atómico Constituyentes, Comisión Nacional de Energía Atómica, \\ Av. Gral Paz 1499, 1650 Gral San Martin, Argentina \\ ${ }^{2}$ Department of Physics, University of La Plata, 671900 La Plata, Argentina \\ ${ }^{3}$ Department of Physics, University of Jyväskylä, P.O. Box 35, FI-40014 Jyväskylä, Finland \\ (Received 21 March 2011; revised manuscript received 18 July 2012; published 28 August 2012)
}

\begin{abstract}
The excitation of Gamow-Teller (GT) and isovector spin monopole (IVSM) $J^{\pi}=1^{+}$modes in ${ }^{116} \operatorname{In}$ by $(p, n)$ and $(n, p)$ charge-exchange reactions on ${ }^{116} \mathrm{Cd}$ and on ${ }^{116} \mathrm{Sn}$, respectively, is studied within the framework of the quasiparticle random-phase approximation. The calculations have been performed both for schematic and realistic model situations. It appears that the calculated admixture of the IVSM and Gamow-Teller (GT) $J^{\pi}=1^{+}$ excitations is negligible and that the contribution to the strength above $20 \mathrm{MeV}$ of excitation energy, in ${ }^{116} \mathrm{In}$, is due to the IVSM $\left(\sigma r^{2} t^{ \pm}\right)$mode. This result is compared with the recent experimental work that reported a large amount of both $(p, n)$ and $(n, p)$ strength beyond $10 \mathrm{MeV}$ of excitation energy in ${ }^{116} \mathrm{In}$. This measured excess strength has led to speculations about its importance for the double beta decay rate of ${ }^{116} \mathrm{Cd}$.
\end{abstract}

DOI: 10.1103/PhysRevC.86.024314

PACS number(s): 24.30.Cz, 21.60.-n, 25.40.Hs, 27.60.+j

\section{INTRODUCTION}

The $(p, n)$ and $(n, p)$ charge-exchange reactions can be used to construct the $\mathrm{t}^{-}\left(\beta^{-}\right)$and $\mathrm{t}^{+}\left(\beta^{+}\right)$strength functions in odd-odd nuclei starting from their neighboring even-even isobars [1]. Recently, the use of these strength functions, to reconstruct the nuclear matrix elements (NMEs) of the twoneutrino double beta $(2 v \beta \beta)$ decays in several nuclei, was carefully analyzed [2]. Similar arguments were pursued by Sasano and coworkers [3,4], in a series of measurements of Gamow-Teller (GT) strengths in $(p, n)$ and $(n, p)$ reactions on medium-heavy nuclei.

In the work of $[3,4]$, the analysis of the double differential cross section for the ${ }^{116} \mathrm{Cd}(p, n)$ and ${ }^{116} \operatorname{Sn}(p, n)$ reactions has been performed by applying a multipole decomposition technique to extract the GT strengths in the intermediate nucleus ${ }^{116} \mathrm{In}$, including the contributions from the possible excitation of IVSM modes. From the theoretical calculations accompanying the analysis of [4] the authors have concluded that the measured strength in the $\beta^{-}$direction has been qualitatively reproduced, except for energies above the GT giant resonance region, and that half of the measured strength in the $\beta^{+}$direction can be accounted for by the theoretical calculations, which include the IVSM mode. These findings are relevant for the reconstruction of the nuclear matrix element governing the two-neutrino double beta decay of ${ }^{116} \mathrm{Cd}$. Such reconstruction, if successful, can help in constraining the parameter spaces of different nuclear models aiming at calculations of the $2 v \beta \beta$ NMEs [5]. Needless to say that such constraints pave the way to a more reliable determination of the NMEs related to the neutrinoless double beta $(0 \nu \beta \beta)$ decays. The observation of the $0 v \beta \beta$ decay will have tremendous consequences upon our present knowledge of the leptonic sector of the electroweak theory, as discussed, e.g., in [5-10].

As mentioned above, a critical issue about $0 \nu \beta \beta$ decays is the need of reliable NMEs because without them the value of the electron-neutrino mass cannot be determined. A suitable testing ground of the related model calculations is the $2 v \beta \beta$ NMEs that can also be accessed by the available $2 v \beta \beta$ half-life measurements and the charge-exchange reactions mentioned earlier.

In this paper we want to address, in particular, the work of [4], where the results of measurements of the $(p, n)$ and $(n, p)$ charge-exchange reactions on ${ }^{116} \mathrm{Cd}$ and ${ }^{116} \mathrm{Sn}$, respectively, have been analyzed. The measurements performed by Sasano et al. [4] were aimed at studying the distributions of Gamow-Teller transition strengths in ${ }^{116} \mathrm{In}$, that is in the odd-odd nucleus which mediates the double beta decay of ${ }^{116} \mathrm{Cd}$.

The analysis of the data shows that (i) the dominant contribution to the cross section $\sigma_{\mathrm{GT}}$ for the ${ }^{116} \mathrm{Cd}(p, n)^{116} \mathrm{In}$ reaction is due to $\Delta l=0$ transitions around the energy of the Gamow-Teller giant resonance (GTGR); (ii) a considerable amount of strength lies at energies larger than the energy of the GTGR; (iii) the multipole decomposition (MD) analysis performed in [4] (see Fig. 1 of [4]) cannot distinguish between pure GT transitions and other transitions with $\Delta l=0$, like those due to the excitation of $\sigma r^{2} \mathrm{t}^{ \pm}$modes; and (iv) most of the strength, at energies above the GTGR, results from the sum of $\Delta l=0$ and 2 , transitions. The same features emerge from the MD analysis of the ${ }^{116} \operatorname{Sn}(n, p)^{116} \mathrm{In}$ reaction. According to [4] a relatively large amount of strength, some 11 units of $\mathrm{B}(\mathrm{GT})$ in the $\beta^{+}$side of the transitions, was found in the energy region below $30 \mathrm{MeV}$. This result has some implications for theoretical double beta decay studies, because it suggests that a sizable contribution to the NME may come from states lying at and above the energy of the GTGR. Taking the results of Sasano et al. [3,4] as a motivation, we shall explore here the excitation of isovector spin monopole (IVSM) states as a possible mechanism to be considered to explain the presence of a relatively large number of spin-dependent transitions at those energies. Since the selection rules of allowed GT transitions exclude transitions induced by tensor operators, we shall restrict the study to $\sigma r^{2} \mathrm{t}^{ \pm}$modes (that is, $\mathrm{IVSM}^{ \pm}$ modes) and study their competition with the allowed $\sigma \mathrm{t}^{ \pm}$ 


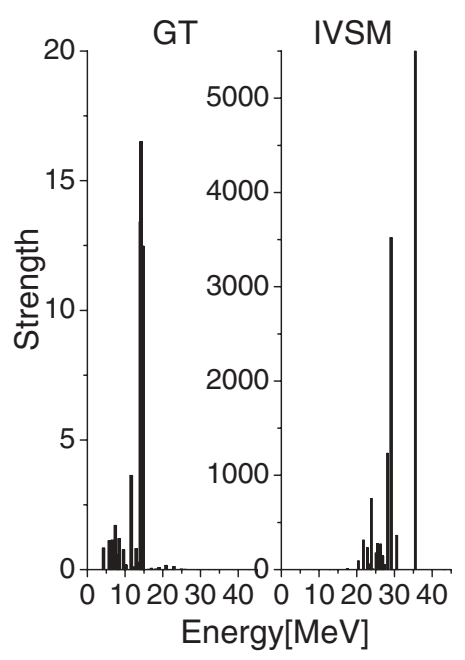

FIG. 1. Distribution of the $\mathrm{GT}^{-}$(left) and $\mathrm{IVSM}^{-}$(right) strengths in the nondegenerate single-particle space with separable proton-neutron interactions. The horizontal axes give the excitation energy in ${ }^{116} \mathrm{In}$. The IVSM strength is given in units of $\mathrm{fm}^{4}$.

GT modes (that is, $\mathrm{GT}^{ \pm}$modes). We shall focus on (i) the strength distributions resulting from the excitation of both the $\mathrm{GT}^{ \pm}$and $\mathrm{IVSM}^{ \pm}$modes and (ii) the competition between these modes in building the $(n, p)$ and $(p, n)$ strengths seen experimentally.

The occurrence of the IVSM has been studied previously by Hamamoto and Sagawa [11]. The authors of [11] have used a self-consistent Hartree-Fock plus Tamm-Dancoff approximation, with a Skyrme-type interaction, to calculate the responses of ${ }^{48} \mathrm{Ca},{ }^{90} \mathrm{Zr}$, and ${ }^{208} \mathrm{~Pb}$ to charge-exchange spin-dependent monopole fields. In doing so, they have separated the GT strength from the IVSM strength. They found that the IVSM strength is much larger than the GT one, in the $t^{-}$side of the transitions [that is, the one seen in $(p, n)$ reactions], and that the centroid of both modes are well separated. The energy of the $\mathrm{IVSM}^{-}$mode was calculated at approximately $35 \mathrm{MeV}$, for the case of ${ }^{90} \mathrm{Zr}$, a nucleus which is not very far away from the $A=116$ mass region and that can be taken as a reference for the energetics of the $\mathrm{IVSM}^{-}$. However, the calculated energies of the $\mathrm{IVSM}^{-}$mode, for the other cases considered in [11], are nearly independent of the mass number.

In the present work we have adopted some of the previously advanced notions [11,12] about GT and IVSM excitations to formulate a schematic model treatment of GT and IVSM excitations in order to understand the competition between them. This is needed in view of the limitation of the MD analysis, since both excitations may contribute to $\Delta l=0$ transitions. As the next step we have performed a realistic model calculation of the transitions, to compare the theoretical results with the strength found in the reactions reported in $[3,4]$.

The adopted framework, for the realistic calculations, is the quasiparticle random-phase approximation (QRPA). The calculations are performed in a proton and neutron singleparticle basis which includes all states up to the $N \leqslant 6$ major shell. Though the basis does not include single-particle states in the continuum, something which is certainly needed if one aims at the calculation of the strength distribution of the IVSM $^{-}$mode at very high energies (as done for instance in [11]), it is large enough to yield a good description of both branches of the GT excitations and the strength of the IVSM ${ }^{+}$ mode. Also, a sizable portion of the strength of the $\mathrm{IVSM}^{-}$ mode is captured in the calculations.

In the present paper we aim at the study of the interplay between the two types of modes, the $\mathrm{GT}^{ \pm}$and the $\mathrm{IVSM}^{ \pm}$ ones in the energy domain $E \leqslant 30 \mathrm{MeV}$ relevant for the bulk of the GT type of strength. In particular we are interested in the identification of the $\mathrm{GT}^{ \pm}$and $\mathrm{IVSM}^{ \pm}$strengths around and above the GTGR energy. The adopted single-particle basis suffices for this purpose, since in it (i) the energetics and sum rules of the $\mathrm{GT}^{ \pm}$modes are strictly reproduced and (ii) the distributions of strength associated to the $\mathrm{IVSM}^{ \pm}$ modes are correctly described for energies $E \leqslant 30 \mathrm{MeV}$ relevant for the comparison with the $(p, n)$ and $(n, p)$ results of [4].

In the next section, Sec. II, we introduce the essentials of the formalism, both for the schematic, Sec. II A, and for the realistic, Sec. II C, model situations. In Sec. III we present and discuss the results of the calculations. Finally, in Sec. IV we summarize and give our conclusions.

\section{FORMALISM}

The allowed isovector spin-dependent operators, which induce the correlations and transitions we are interested in, are the Gamow-Teller $\left(\sigma \mathrm{t}^{ \pm}\right)$and the isovector spin monopole $\left(\sigma r^{2} \mathrm{t}^{ \pm}\right)$operators. Their effects have been studied in connection with charge-exchange reactions on ${ }^{90} \mathrm{Zr}$, both theoretically [11] and experimentally [13,14]. In this section we shall further extend the study of [11] to determine the amount of interplay between the GT and IVSM excitations at some extreme limits, namely, (i) in a single-particle model space consisting of degenerate oscillator shells, (ii) with separable interactions in a nondegenerate single-particle space, and (iii) in a realistic situation. In the following subsections we shall study, analytically, the possible coupling between the GT and IVSM modes and the effects of these possible couplings on the response functions of each mode.

\section{A. Extreme single-particle model space}

Let us assume that there is a vacuum state on top of which we may perform the excitations:

$$
\gamma_{N^{\prime} l^{\prime} j^{\prime} ; N l j ; M}^{+}=\left[b_{N^{\prime} l^{\prime} j^{\prime}}^{+} c_{N l j}\right]_{M}^{1} .
$$

We deal with isovector moments carrying angular momentum 1 and angular-momentum projection $M$, and positive parity. The moments which annihilate a neutron $\left(c_{N l j}\right)$ and create a proton $\left(b_{N^{\prime} l^{\prime} j^{\prime}}^{+}\right)$are written

$$
\begin{aligned}
Q_{\nu \lambda, M}= & \sum_{N^{\prime} l^{\prime} j^{\prime}, N l j} q_{N^{\prime} l^{\prime} j^{\prime} ; N l j}^{(\nu \lambda)}\left(\gamma_{N^{\prime} l^{\prime} j^{\prime} ; N l j ; M}^{+}\right. \\
& \left.+(-1)^{M} \gamma_{N^{\prime} l^{\prime} j^{\prime} ; N l j ; \bar{M}}\right),
\end{aligned}
$$


where

$$
q_{N^{\prime} l^{\prime} j^{\prime} ; N l j}^{(\nu \lambda)}=-\frac{1}{\sqrt{3}}\left\langle N^{\prime} l^{\prime}\left|r^{\nu}\right| N l\right\rangle\left\langle\left[Y_{l^{\prime}} s\right]^{j^{\prime}}\left\|\left[Y_{\lambda} \sigma\right]^{1}\right\|\left[Y_{l} s\right]^{j}\right\rangle .
$$

The indexes $(\nu \lambda)$ of Eq. (3) may take the values $(\nu=2, \lambda=$ $2),(\nu=2, \lambda=0)$, and $(v=0, \lambda=0)$, respectively. The pair (00) is associated to the GT operator $\sigma t^{ \pm}$, the pair $(2,0)$ to the operator $\sigma r^{2} t^{ \pm}$, and the pair (22) to the tensor $\left(\sigma Y_{2}\right)_{1} r^{2} t^{ \pm}$. The single-particle model space is then specified by the principal quantum number $(N)$, the orbital angular momentum $(l)$, and the total angular momentum $(j)$.

Considering the selection rules of the operators (2), there are three possible excitations within our space:

$$
\begin{aligned}
& \text { (i) } N^{\prime}=N, N+2 ; \quad l^{\prime}=l \pm 2, \\
& \text { (ii) } N^{\prime}=N+2 ; \quad l^{\prime}=l, \\
& \text { (iii) } N^{\prime}=N ; \quad l^{\prime}=l,
\end{aligned}
$$

and $j^{\prime}=j, j \pm 1$ in all cases.

While the moment labeled by $Q_{22, M}$, which in this extreme model corresponds to the isovector spin-quadrupole operator $\left(\sigma Y_{2}\right)_{1} r^{2} t^{ \pm}$, may include the three type of transitions (i)-(iii) (with $\Delta l=0, \pm 2$, and $\Delta N=0,2$ ), the multipole moment $Q_{20, M}$ can accommodate transitions (ii) and (iii) (both with $\Delta l=0$, and for $\Delta N=0,2$ ), and the moment $Q_{00, M}$ activates only transitions (iii) (for which $\Delta l=\Delta N=0$ ). As said before, for all cases $j^{\prime}=j, j \pm 1$.

To study the correlations between the modes, we introduce, in a first step, the separable interactions

$$
H_{\nu \lambda}=\frac{\chi_{\nu \lambda}}{2} \sum_{M}(-1)^{M} Q_{\nu \lambda, M} Q_{\nu \lambda, \bar{M}},
$$

and, in a second step, we evaluate their mixing by using perturbation theory. Notice that to perform the first step we take the two multipole moments [the (22) and (20) moments] which share the $\Delta N=2$ space, while the (00) moment lives only in the $\Delta N=0$ space. Thus, perturbation theory will be applied to couple both spaces analytically.

To proceed with the calculations, (i) we restrict our space to the degenerate excitations with $N^{\prime}=N+2$ [therefore, with the unperturbed energy $E_{0}(\Delta N=2)=\Delta E=2 \hbar \omega$ for all these excitations], to construct the configurations for the (22) and (20) modes [thus the total Hamiltonian for this subspace will be $\left.H=h_{0}(\Delta N=2)+H_{22}+H_{20}\right]$; (ii) we treat the $\Delta N=0$ excitations with $l^{\prime}=l=N$, with unperturbed energy $E_{0}(\Delta N=0)=\epsilon_{0}$, which is the spin-orbit splitting within the shell, to construct the space of the (00) mode [thus the total Hamiltonian for this subspace will be $H=h_{0}(\Delta N=0)+$ $\left.H_{00}+H_{22}(\Delta N=0)+H_{20}(\Delta N=0)\right]$; and (iii) we couple both spaces within perturbation theory in order to obtain the matrix element of the $Q_{00, M}$ operator populating $\Delta N=2$ states.

In all cases we shall consider a fixed value of $N$, all allowed values of $l$ within the shell specified by $N$, and all values of $j$ allowed by the selection rules of the operators $Q_{\nu \lambda}$. In the following equations all summations will then run over the quantum numbers $l$ and $j$, and we shall omit summation indices unless they are needed for the sake of clarity.
The uncoupled phonons in the $\Delta N=2$ subspace $^{1}$ are given by the RPA transformation

$$
\begin{aligned}
\Gamma_{2 n, M}^{+}= & \sum_{l j j^{\prime}}\left[\left(\lambda_{2 n ;(N+2)(l \pm 2) j^{\prime} ; N l j} \gamma_{(N+2)(l \pm 2) j^{\prime} ; N l j ; M}^{+}\right.\right. \\
& \left.+\lambda_{2 n ;(N+2) l j^{\prime} ; N l j} \gamma_{(N+2) l j^{\prime} ; N l j ; M}^{+}\right) \\
& -(-1)^{M}\left(\mu_{2 n ;(N+2)(l \pm 2) j^{\prime} ; N l j} \gamma_{(N+2)(l \pm 2) j^{\prime} ; N l j ; \bar{M}}\right. \\
& \left.+\mu_{2 n ;(N+2) l j^{\prime} ; N l j} \gamma_{(N+2) l j^{\prime} ; N l j ; \bar{M}}\right] .
\end{aligned}
$$

The amplitudes of Eq. (6) are determined from the RPA linearization procedure. The multipole moments (2) satisfy the commutation relations

$$
\begin{aligned}
{\left[Q_{2 \lambda, \bar{M}}, \Gamma_{2 n, M}^{+}\right]=} & (-1)^{M} \Lambda_{2 n ; 2 \lambda}, \\
\Lambda_{2 n ; 22}= & \sum_{l j j^{\prime}}\left(\lambda_{2 n ;(N+2)(l \pm 2) j^{\prime} ; N l j}+\mu_{2 n ;(N+2)(l \pm 2) j^{\prime} ; N l j}\right) \\
& \times q_{(N+2)(l \pm 2) j^{\prime} ; N l j}^{(22)}+\left(\lambda_{2 n ;(N+2) l j^{\prime} ; N l j}\right. \\
& \left.+\mu_{2 n ;(N+2) l j^{\prime} ; N l j}\right) q_{(N+2) l j^{\prime} ; N l j}^{(22)}, \\
\Lambda_{2 n, 20}= & \sum_{l j j^{\prime}}\left(\lambda_{2 n ;(N+2) l j^{\prime} ; N l j}+\mu_{2 n ;(N+2) l j^{\prime} ; N l j}\right) \\
& \times q_{(N+2) l j^{\prime} ; N l j}^{(20)} .
\end{aligned}
$$

The amplitudes appearing in Eq. (6) are then written

$$
\begin{aligned}
\lambda_{2 n ;(N+2)(l \pm 2) j^{\prime} ; N l j}= & \chi_{22} \Lambda_{2 n ; 22} q_{(N+2)(l \pm 2) j^{\prime} ; N l j}^{(22)} /\left(2 \hbar \omega-W_{2 n}\right), \\
\lambda_{2 n ;(N+2) l j^{\prime} ; N l j}= & \left(\chi_{22} \Lambda_{2 n ; 22} q_{(N+2) l j^{\prime} ; N l j}^{(22)}\right. \\
& \left.+\chi_{20} \Lambda_{2 n ; 20} q_{(N+2) l j^{\prime} ; N l j}^{(20)}\right) /\left(2 \hbar \omega-W_{2 n}\right), \\
\mu_{2 n ;(N+2)(l \pm 2) j^{\prime} ; N l j}= & \chi_{22} \Lambda_{2 n ; 22} q_{(N+2)(l \pm 2) j^{\prime} ; N l j}^{(22)} /\left(2 \hbar \omega+W_{2 n}\right), \\
\mu_{2 n ;(N+2) l j^{\prime} ; N l j}= & \left(\chi_{22} \Lambda_{2 n ; 22} q_{(N+2) l j^{\prime} ; N l j}^{(22)}\right. \\
& \left.+\chi_{20} \Lambda_{2 n ; 20} q_{(N+2) l j^{\prime} ; N l j}^{(20)}\right) /\left(2 \hbar \omega+W_{2 n}\right) .
\end{aligned}
$$

The energies $W_{ \pm}$of the correlated states, associated with the two modes resulting from the two channels $(\nu \lambda)=(22),(20)$ of Eq. (5), can be determined from self-consistency, which yields the eigenvalue equation

$$
\left(\chi_{22} A_{22}+B_{ \pm}\right)\left(\chi_{20} A_{20}+B_{ \pm}\right)=\chi_{22} \chi_{20} C^{2},
$$

with the solutions

$$
W_{ \pm}^{2}=4 \hbar^{2} \omega^{2}-4 \hbar \omega B_{ \pm},
$$

where

$$
\begin{aligned}
B_{ \pm}= & -\frac{1}{2}\left(\chi_{22} A_{22}+\chi_{20} A_{20}\right) \\
& \left. \pm \frac{1}{2}\left(\chi_{22} A_{22}-\chi_{20} A_{20}\right)^{2}+4 \chi_{22} \chi_{20} C^{2}\right)^{1 / 2},
\end{aligned}
$$

\footnotetext{
${ }^{1} n=0,2$ indicates the two isovector spin modes which can operate in this subspace.
} 
and

$$
\begin{aligned}
A_{22} & =\sum\left(\left(q_{(N+2)(l \pm 2) j^{\prime} ; N l j}^{(22)}\right)^{2}+\left(q_{(N+2) l j^{\prime} ; N l j}^{(22)}\right)^{2}\right), \\
A_{20} & =\sum\left(q_{(N+2) l j^{\prime} ; N l j}^{(20)}\right)^{2}, \\
C & =\sum q_{(N+2) l j^{\prime} ; N l j}^{(22)} q_{(N+2) l j^{\prime} ; N l j}^{(20)} .
\end{aligned}
$$

Equations (10)-(12) are indeed coupled equations, and the coupling between the two modes which are operative in the $\Delta N=2$ space is given by the term in the right-hand side of Eq. (9).

The summations of Eq. (12) run over $l, j$, and $j^{\prime}$. A priori, the coupling between the $\Delta N=2$ phonons offers the possibility of a large enhancement to the state with the appropriate phase. However, let us examine the value of the coupling parameter $C$, which is given by the expression

$$
C=\left\langle\frac{1}{2}\|\sigma\| \frac{1}{2}\right\rangle \sum_{l}\left\langle(N+2) l\left|r^{2}\right| N l\right\rangle^{2}\left\langle l\left\|Y_{2}\right\| l\right\rangle\left\langle l\left\|Y_{00}\right\| l\right\rangle S,
$$

where $S$ is a sum of products of $9 j$ symbols:

$$
S=\sum_{j^{\prime} j}(2 j+1)\left(2 j^{\prime}+1\right)\left\{\begin{array}{ccc}
l & l & 2 \\
\frac{1}{2} & \frac{1}{2} & 1 \\
j & j^{\prime} & 1
\end{array}\right\}\left\{\begin{array}{ccc}
l & l & 0 \\
\frac{1}{2} & \frac{1}{2} & 1 \\
j & j^{\prime} & 1
\end{array}\right\},
$$

which vanishes because of the orthogonality relations between the $9 j$ symbols. Therefore, the two $\Delta N=2$ phonons are uncoupled in the limit of degenerate $2 \hbar \omega$ single-particle excitations. Their energy and specific operator are

$$
\begin{aligned}
W_{2 \lambda} & =2 \hbar \omega\left(1+\chi_{2 \lambda} A_{2 \lambda} / \hbar \omega\right)^{1 / 2}, \\
Q_{2 \lambda, M} & =\Lambda_{2 \lambda}\left(\Gamma_{2 \lambda, M}^{+}+(-1)^{M} \Gamma_{2 \lambda, \bar{M}}\right), \quad \Lambda_{2 \lambda}=A_{2 \lambda} 2 \hbar \omega / W_{2 \lambda} .
\end{aligned}
$$

Since the ratio $A_{22} / A_{20}$ is close to unity, the relative properties of the $\Delta N=2$ states are determined by the ratio $\chi_{22} / \chi_{20}$.

Let us consider now the $(\Delta N=0, \Delta l=0, \Delta j=0, \pm 1)$ excitations, which correspond to the radial-independent Gamow-Teller operator. The GT phonon is written

$$
\begin{aligned}
\Gamma_{0, M}^{+}= & \sum_{l j j^{\prime}}\left(\lambda_{0 ; N l j^{\prime} ; N l j} \gamma_{N l j^{\prime} ; N l j ; M}^{+}\right. \\
& \left.+(-1)^{M} \mu_{0 ; N l j^{\prime} ; N l j} \gamma_{N l j^{\prime} ; N l j ; \bar{M}}\right) .
\end{aligned}
$$

The commutation with the GT one-phonon operator gives

$$
\begin{aligned}
{\left[Q_{\nu \lambda ; \bar{M}}, \Gamma_{0, M}^{+}\right] } & =(-1)^{M} \Lambda_{0 ; \nu \lambda}, \\
\Lambda_{0 ; \nu \lambda} & =\sum_{l j j^{\prime}} q_{N l j^{\prime} ; N l j}^{(\nu \lambda)}\left(\lambda_{0 ; N l j^{\prime} ; N l j}+\mu_{0 ; N l j^{\prime} ; N l j}\right) .
\end{aligned}
$$

We obtain the amplitudes

$$
\begin{aligned}
& \lambda_{0 ; N l j^{\prime} ; N l j}=-\sum_{\nu \lambda} \chi_{\nu \lambda} \Lambda_{0 ; \nu \lambda} q_{N l j^{\prime} ; N l j}^{(\nu \lambda)} /\left(\epsilon_{0}-W_{0}\right), \\
& \mu_{0 ; N l j^{\prime} ; N l j}=-\sum_{\nu \lambda l j j^{\prime}} \chi_{\nu \lambda} \Lambda_{0 ; \nu \lambda} q_{N l j^{\prime} ; N l j}^{(\nu \lambda)} /\left(\epsilon_{0}+W_{0}\right),
\end{aligned}
$$

and the energy $W_{0}$ :

$$
W_{0}^{2}=\epsilon_{0}^{2}+2 \epsilon_{0} \sum_{\nu \lambda, l} \chi_{\nu \lambda}\left(q_{N l j^{\prime} ; N l j}^{(\nu \lambda)}\right)^{2} .
$$

Since the ratio between the phonon-strength functions $\Lambda_{0 ; 2 \lambda} / \Lambda_{0,00}$ is fixed by the system of Eqs. (18), the normalization condition yields

$$
\begin{aligned}
1 & =\sum_{l j j^{\prime}} \lambda_{0 ; N l j^{\prime} ; N l j}^{2}-\mu_{0 ; N l j^{\prime} ; N l j}^{2} \\
& =\left(\sum_{\nu \lambda l j j^{\prime}} \chi_{\nu \lambda} \Lambda_{0 ; \nu \lambda} q_{N l j^{\prime} ; N l j}^{(\nu \lambda)}\right)^{2} \frac{4 \epsilon_{0} W_{0}}{\left(\epsilon_{0}^{2}-W_{0}^{2}\right)^{2}} \\
& =\left(\frac{\Lambda_{0 ; 00}}{\sum_{l j j^{\prime}} q_{N l j^{\prime} ; N l j}^{(00)}}\right)^{2} \frac{W_{0}}{\epsilon_{0}},
\end{aligned}
$$

and, from this normalization condition we can extract the values of $\Lambda_{0 ; 00}$ and $\Lambda_{0 ; 2 \lambda}$.

Finally, to fulfill step (iii), we are interested in the transition amplitude of the moment $Q_{00, M}$, to the $\Delta N=2$ excitations. It can be calculated perturbatively, and the result is given by the expression

$$
\frac{\left\langle\Gamma_{2 \lambda, M}^{+}\left|Q_{0 ; M}\right| \text { g.s. }\right\rangle}{\left\langle\Gamma_{0, M}^{+}\left|Q_{0 ; M}\right| \text { g.s. }\right\rangle}=\frac{\sum_{\lambda} \chi_{2 \lambda} \Lambda_{0,2 \lambda} \Lambda_{2 \lambda}}{W_{2 \lambda}-W_{0}} .
$$

To write Eq. (21) we have used Eqs. (7) and (18), where the couplings are expressed within the QRPA formalism, by inversion of the phonons, that is, by writing the quasiparticle pairs in terms of phonons. Therefore, for this extreme singleparticle model, it is expected that the admixture between the GT and IVSM modes is strongly suppressed, because of the large energy denominator appearing in Eq. (21). In fact, the energy denominator is of the order of $2 \hbar \omega \approx 12-14 \mathrm{MeV}$, and it means that the expected admixture of the IVSM mode in the wave function of the GT mode cannot be larger than, say, a few percent, even if the value of the numerator of Eq. (21) is replaced by unity.

\section{B. Nondegenerate single-particle space}

If the single-particle space is nondegenerate we have to solve the RPA equations for all possible configurations of proton-neutron pairs. Furthermore, since we are dealing with open shells, we have to include pairing occupation factors in our equations, which will then be written in the form of the standard proton-neutron (pn) QRPA equations [5]. Each of the amplitudes $\lambda_{p n}(k J)$ and $\mu_{p n}(k J)$ are associated with the excitation energy $W_{k}(J)$ of the $k$ th state with angular momentum and parity $J^{\pi}=1^{+}$. They are the eigenvectors and eigenvalues, respectively, of the equations

$$
\left(\begin{array}{ll}
A(J) & B(J) \\
B^{*}(J) & A^{*}(J)
\end{array}\right)\left(\begin{array}{l}
\lambda(k J) \\
\mu(k J)
\end{array}\right)=W_{k}(J)\left(\begin{array}{l}
\lambda(k J) \\
-\mu(k J)
\end{array}\right),
$$


where the matrix elements of the pnQRPA matrix Eq. (22) are given by

$$
A_{p n, p^{\prime} n^{\prime}}=\left\langle 0\left|\left[\gamma_{p n},\left[H, \gamma_{p^{\prime} n^{\prime}}^{\dagger}\right]\right]\right| 0\right\rangle, \quad B_{p n, p^{\prime} n^{\prime}}=-\left\langle 0\left|\left[\gamma_{p n} .\left[H, \gamma_{p^{\prime} n^{\prime}}\right]\right]\right| 0\right\rangle .
$$

For the interaction between protons and neutrons, $H_{p n}$, we adopt the multilevel separable-type interaction for each mode $(\nu \lambda)$ (we are using the same notation as in the previous subsection):

$$
\begin{aligned}
H_{p n}(\nu \lambda)= & \frac{1}{2} \sum_{p n, M}\left\langle p\left\|Q_{\nu \lambda}\right\| n\right\rangle\left\langle p^{\prime}\left\|Q_{\nu \lambda}\right\| n^{\prime}\right\rangle^{*}\left\{\chi_{\nu \lambda}\left(\left[a_{p}^{\dagger} a_{\bar{n}}\right]^{J M}\left[a_{p^{\prime}}^{\dagger} a_{\bar{n}^{\prime}}\right]^{\dagger \overline{J M}}+\left[a_{p}^{\dagger} a_{\bar{n}}\right]^{\dagger \overline{J M}}\left[a_{p^{\prime}}^{\dagger} a_{\overline{n^{\prime}}}\right]^{J M}\right)\right. \\
& \left.-\kappa_{\nu \lambda}\left(\left[a_{p}^{\dagger} a_{\bar{n}}^{\dagger}\right]^{J M}\left[a_{p^{\prime}}^{\dagger} a_{\bar{n}^{\prime}}^{\dagger}\right]^{\dagger \overline{J M}}+\left[a_{p}^{\dagger} a_{\bar{n}}^{\dagger}\right]^{\dagger \overline{J M}}\left[a_{p^{\prime}}^{\dagger} a_{\bar{n}^{\prime}}^{\dagger}\right]^{J M}\right)\right\},
\end{aligned}
$$

to which we add the unperturbed energy of the quasineutron-quasiproton pairs. The Hamiltonian Eq. (24) is the extension of the Hamiltonian Eq. (5) of the previous section to the nondegenerate single-particle space.

Now using the fact that

$$
\begin{aligned}
& {\left[b_{p}^{\dagger} c_{\bar{n}}\right]^{J M}=-\left[u_{p} v_{n} \gamma_{p n}^{\dagger}(J M)+v_{p} u_{n} \gamma_{p n}(\overline{J M})\right]} \\
& {\left[b_{p}^{\dagger} c_{n}^{\dagger}\right]^{J M}=-\left[u_{p} u_{n} \gamma_{p n}^{\dagger}(J M)-v_{p} v_{n} \gamma_{p n}(\overline{J M})\right],}
\end{aligned}
$$

and assuming the quasiboson approximation

$$
\left[\gamma_{p n}, \gamma_{p^{\prime} n^{\prime}}^{\dagger}\right]=\delta_{p p^{\prime}} \delta_{n n^{\prime}},
$$

we get, for each multipole moment $Q_{\nu \lambda}$, the components of the pnQRPA matrix:

$$
\begin{aligned}
A_{p n, p^{\prime} n^{\prime}}(J)= & \left(E_{p}+E_{n}\right)+\chi_{\nu \lambda}\left\langle p\left\|Q_{\nu \lambda}\right\| n\right\rangle\left\langle p^{\prime}\left\|Q_{\nu \lambda}\right\| n^{\prime}\right\rangle^{*}\left(u_{p} v_{n} u_{p} v_{n}+v_{p} u_{n} v_{p} u_{n}\right) \\
& -\kappa_{\nu \lambda}\left\langle p\left\|Q_{\nu \lambda}\right\| n\right\rangle\left\langle p^{\prime}\left\|Q_{\nu \lambda}\right\| n^{\prime}\right\rangle^{*}\left(u_{p} u_{n} u_{p} u_{n}+v_{p} v_{n} v_{p} v_{n}\right), \\
B_{p n, p^{\prime} n^{\prime}}(J)= & \chi_{\nu \lambda}\left\langle p\left\|Q_{\nu \lambda}\right\| n\right\rangle\left\langle p^{\prime}\left\|Q_{\nu \lambda}\right\| n^{\prime}\right\rangle^{*}\left(v_{p} u_{n} u_{p} v_{n}+u_{p} v_{n} v_{p} u_{n}\right) \\
& -\kappa_{\nu \lambda}\left\langle p\left\|Q_{\nu \lambda}\right\| n\right\rangle\left\langle p^{\prime}\left\|Q_{\nu \lambda}\right\| n^{\prime}\right\rangle^{*}\left(u_{p} u_{n} v_{p} v_{n}+v_{p} v_{n} u_{p} u_{n}\right) .
\end{aligned}
$$

In the previous equations $u_{p}\left(u_{n}\right)$ and $v_{p}\left(v_{n}\right)$ are the BCS occupation factors, and $E_{p}\left(E_{n}\right)$ are the corresponding quasiparticle energies. After performing the diagonalization of the pnQRPA matrix Eq. (27), we have calculated the corresponding strength distribution for each multipole moment, by computing the matrix elements of the multipole moments on the pnQRPA eigenstates. The results are shown in Sec. III.

\section{Realistic calculations}

In order to confront the information which is extracted from the schematic models of the previous section, we have developed a similar framework but based on the use of realistic interactions in realistic single-particle spaces. We have applied the equations of the pnQRPA to solve for the wave functions of the $J^{\pi}=1^{+}$states in ${ }^{116} \mathrm{In}$. We have then calculated the strengths for transitions to those states from the ground state of ${ }^{116} \mathrm{Cd}$ and ${ }^{116} \mathrm{Sn}$. The pnQRPA formalism for the realistic case has been presented at length in many standard texts and we shall refer the reader to [5] for further details. Here we shall summarize the main aspects of the formalism in connection with the description of the GT and IVSM excitations.

As a first step we construct a realistic nuclear two-body interaction. For this we use the Bonn-A potential, with the parameters given in [15], solve for the $G$ matrix at the one-loop level, and add corrections to account for the finite size of the system. Next, we construct the matrix elements of the nuclear two-body interaction in the various channels which are required for the calculations, including monopole-pairing terms. The single-particle basis, needed to calculate the proton- neutron configurations entering the pnQRPA model, was then constructed by using a Coulomb-corrected Woods-Saxon potential with the parametrization of [16]. Finally, we have solved the pnQRPA equations in the manner described in [5]. The outcome of these calculations is a complete set of wave functions and excitation energies for the $J^{\pi}=1^{+}$states in ${ }^{116} \mathrm{In}$. These wave functions were in turn used to calculate the associated GT and IVSM transitions, as described in the following section.

\section{RESULTS AND DISCUSSION}

\section{A. Results of the schematic model}

\section{Degenerate single-particle states within a shell}

For the schematic model situation of Sec. II A (degenerate single-particle states within a shell) we have chosen singleparticle states with principal quantum numbers $N=2$ to 6 . The value of $\epsilon_{0}$ of Eq. (19) was taken from the observed spin-orbit splitting in the $N=4$ and 5 shells, and the ratio between the coupling constants $\chi_{22}$ and $\chi_{20}$ was fixed to bring the energy of the IVSM mode to the region suggested by the study of [11]. Equation (10) yields two solutions, at 16.89 and $25.40 \mathrm{MeV}$, with practically all of the strength concentrated in the higher-energy solution. As predicted by Eqs. (12) there is no mixing between the states since $C$ vanishes, a condition which is fulfilled provided that for each value of $l$ both members of the spin-orbit doublets are included in the single-particle basis. Also, in this degenerate single-particle space, the GT strength is concentrated in the state with energy 
$W_{0}=\epsilon_{0}$ (since the Coulomb shift between proton and neutron states is not considered in the model), and the contribution to it due to the $W_{ \pm}$states is very small, as predicted by Eq. (21).

\section{Nondegenerate single-particle states}

Naturally, we may ask if these features remain when the degeneracy within the shells is removed, like the case of Sec. II B. To answer this question we have performed calculations, for $A=116$, using a basis which consists of 26 single-particle levels for neutrons and protons. The basis includes all states from $N=0$ to 6 major shells; only bound states have been included in the basis, both for protons and neutrons.

The energies of the single-particle states are those given by the parametrization of the harmonic oscillator plus spin-orbit potential of [16]. Monopole pairing interactions were accounted for by solving the BCS formalism with couplings adjusted to reproduce the observed odd-even mass differences. The actual values of the couplings are $g_{n}=18 / A \mathrm{MeV}$ for neutrons and $g_{p}=22 / A \mathrm{MeV}$ for protons. The couplings of the separable proton-neutron interaction were fixed at $\chi=0.12 \mathrm{MeV}$ and $\kappa=0.05 \mathrm{MeV}$ for the $(\nu \lambda)=(0,0)$ mode and $\chi /\left\langle r^{2}\right\rangle_{\text {excess }}^{2}$ and $\kappa=0.0 \mathrm{MeV}$ for the $(\nu \lambda)=(2,0),(2,2)$ modes, respectively. Note that the only adjustable parameters are the couplings $\chi$ and $\kappa$, of the GT mode, and once they are fixed the couplings of the IVSM mode are these quantities scaled by the square of the excess radius $\left\langle r^{2}\right\rangle_{\text {excess }}$ [see Eq. (29)].

After solving the pnQRPA Eqs. (22) with this set of parameters we have obtained a distribution of the $\mathrm{GT}^{-}$ intensity shown in Fig. 1 (left side). The distribution of intensity for the IVSM mode, obtained by solving a similar set of equations with the radial-dependent moments $Q_{20}$ and $Q_{22}$, is shown in Fig. 1 (right side). The intensities are defined as the square of the amplitudes $<1^{+}\left\|O \mathrm{t}^{ \pm}\right\| 0^{+}>$, where $O=\sigma$ (GT mode) or $O=\sigma r^{2}$ (IVSM mode). In this way the GT intensities are dimensionless and the IVSM ones are given in units of $\mathrm{fm}^{4}$.

It is seen that the results of the calculations do show the features extracted from the extreme single-particle model, i.e., there is no visible admixture of the $\mathrm{IVSM}^{-}$mode with the radial independent $\mathrm{GT}^{-}$mode. The calculation yields $\omega_{\mathrm{GTGR}}=14.7 \mathrm{MeV}$ and the strength corresponding to $\mathrm{IVSM}^{-}$ transitions is well separated and its centroid is located at about 33-34 MeV. Unlike in the case of the extreme single-particle model, the $\mathrm{GT}^{-}$and $\mathrm{IVSM}^{-}$strengths are split among several states. Not much $\mathrm{GT}^{-}$strength is spread beyond the GTGR.

\section{B. Results of the realistic calculation}

The realistic calculations were performed in exactly the same model space that was used in the nondegenerate schematic model of the previous section. The Woods-Saxon energies of these single-particle states were used along with the Bonn-A two-body potential derived by the $G$-matrix technique. The monopole pairing strength was fixed in a BCS calculation by the odd-even mass differences, separately for protons and neutrons. The overall strength of the particlehole part of the proton-neutron $J^{\pi}=1^{+}$interaction was determined by the empirical location of the centroid of the GTGR state and the strength of the particle-particle part was fixed such that the strength of the transition ${ }^{116} \mathrm{Cd}\left(0^{+}\right) \rightarrow$ ${ }^{116} \operatorname{In}\left(1_{1}^{+}\right)$has a reasonable value.

Before starting with the discussion of the results of the QRPA calculations, we shall comment on the single-particle basis used in the calculations, with reference to the properties of both the GT and IVSM modes. In the adopted basis the Ikeda sum rule is fulfilled for the GT modes. For the IVSM modes the situation is different because we are dealing with bound states only. In order to have an idea about the amount of strength resulting from the excitation of IVSM $^{ \pm}$modes, in the same basis, we have calculated the difference between the intensities of the shifted operators $\mathrm{O}_{1 \mu} \mathrm{t}^{ \pm}=\sigma_{1 \mu}\left(\mathrm{r}^{2}-\left\langle\mathrm{r}^{2}\right\rangle_{\text {excess }}\right) \mathrm{t}^{ \pm}$, where

$$
\begin{aligned}
& \text { Intensity }\left(\mathrm{O}_{1 \mu} \mathrm{t}^{ \pm}\right) \\
& \quad=\sum_{n, \mu}\left|\left\langle 1_{n}^{+}\left|\sigma_{1 \mu}\left(r^{2}-\left\langle r^{2}\right\rangle_{\text {excess }}\right) t^{ \pm}\right| 0^{+}\right\rangle\right|^{2} .
\end{aligned}
$$

The expression

$$
\begin{aligned}
& \text { Intensity }\left(\mathrm{O}^{-}\right)-\operatorname{Intensity}\left(\mathrm{O}^{+}\right) \\
& =3\left(N\left\langle r^{4}\right\rangle_{n}-Z\left\langle r^{4}\right\rangle_{p}\right)+3\left\langle r^{2}\right\rangle_{\text {excess }}^{2}(N-Z) \\
& \quad-6\left\langle r^{2}\right\rangle_{\text {excess }}\left(N\left\langle r^{2}\right\rangle_{n}-Z\left\langle r^{2}\right\rangle_{p}\right)
\end{aligned}
$$

represents the difference between the total strengths carried by each branch of the excitations [11].

The second and third terms of Eq. (29) result from the use of the shifted radial operator $r^{2} \rightarrow\left(r^{2}-\left\langle r^{2}\right\rangle_{\text {excess }}\right)$ [11], and the quantities $\left\langle r^{2}\right\rangle_{n}$ and $\left\langle r^{2}\right\rangle_{p}$ are the values of $r^{2}$ taken over the occupied neutron $\left(\left\langle r^{2}\right\rangle_{n}\right)$ and proton $\left(\left\langle r^{2}\right\rangle_{p}\right)$ single-particle orbits (the corresponding summations included all levels up to the neutron and proton Fermi levels, each level weighted by the corresponding occupation factors), while $\left\langle r^{2}\right\rangle_{\text {excess }}$ is the excess radius (hereby introduced to describe the excess of neutron occupied orbits over the proton occupied orbits and calculated as an average over the neutron excess orbits) [11]. For $\left\langle r^{2}\right\rangle_{\text {excess }}=24 \mathrm{fm}^{2}$, Eq. (29) yields $1.40 \times$ $10^{4} \mathrm{fm}^{4}$ for the intensity difference. ${ }^{2}$ This value is in good agreement with the systematics reported in [11], but it turns out that the corresponding QRPA value, in the basis of bound single-particle states, is smaller than this. However, the missing intensity (attributed to the contributions coming from the continuum) lies at energies above $45 \mathrm{MeV}$. These energies are much larger than the ones where the beta decay is operative.

Returning to our problem, the previous results may suggest that the presence of IVSM strength above $45 \mathrm{MeV}$ may not be relevant for our present analysis, which focuses on a much lower-energy region where the overlap between the GT and IVSM modes may result in the enhancement of the GT transitions.

Because of the selection rules of the IVSM operator, and specifically because of its spin dependence, it is not expected that the inclusion of high-lying single-particle states (either quasibound or unbound) may contribute significantly to the

\footnotetext{
${ }^{2}$ If one takes instead a mean value of $\left\langle r^{2}\right\rangle$, starting from the calculated neutron and proton $\left\langle r^{2}\right\rangle$ values, and for this case $\left\langle r^{2}\right\rangle_{\text {mean }}=$ $21 \mathrm{fm}^{2}$, Eq. (29) yields a slighter larger value, $1.62 \times 10^{4} \mathrm{fm}^{4}$.
} 


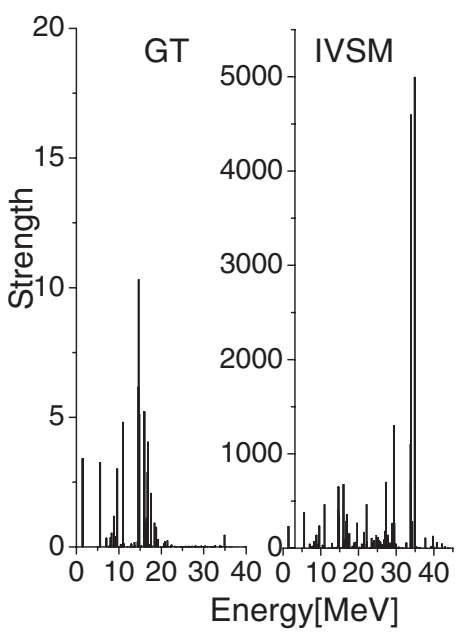

FIG. 2. The same as in Fig. 1 for the realistic calculation. The calculated $\mathrm{GT}^{-}$and $\mathrm{IVSM}^{-}$strengths are given in the same units as in Fig. 1; the IVSM transitions have been calculated by excluding $\Delta N=0$ contributions.

strength at lower energies (spin operators tend to shift the intensity upwards), with the possible exception of isolated resonances from where the nucleus can decay by particle emission. This effect has been verified, for instance, in the calculation of spin-isospin dependent modes in the $A=208$ region [17].

The results for the strength distributions of $\mathrm{GT}^{-}$and IVSM $^{-}$excitations are shown in Fig. 2. To make them comparable we have removed the $\Delta N=0$ contributions to the IVSM mode. The general picture does not differ much from the one obtained with the separable interaction Eq. (24), although there seems to be some more structure, for the IVSM mode, in the region below $30 \mathrm{MeV}$ of excitation. This is a small fraction of the IVSM strength, which is basically concentrated at $34 \mathrm{MeV}$. Also the GTGR is wider for the realistic calculation, spreading strength up to $20 \mathrm{MeV}$ of excitation in ${ }^{116} \mathrm{In}$.

The position of the $\mathrm{IVSM}^{-}$resonance, at about $34 \mathrm{MeV}$, is approximately $19 \mathrm{MeV}$ higher than the GTGR, which lies at about $15 \mathrm{MeV}$. This pattern is in good agreement with the expected systematics of [11]. The shift of the IVSM resonance to higher energies is of the order of $2 \hbar \omega$, something which was anticipated in [4]. However, in contrast to the assumption of [4], the results of our calculation do not show any significant admixture between the GT and the IVSM modes. The computed amount of $\mathrm{GT}^{-}$strength within the energy range $10 \leqslant E \leqslant 30 \mathrm{MeV}$ amounts to 47 units and is thus larger than the strength measured in [4] below $30 \mathrm{MeV}$ of excitation. Because of the decoupling between the GT and IVSM modes (see the discussion in Sec. II A) the admixture between them is not expected to have a sizable effect upon the strength distributions, unless additional interactions between the modes are included in the Hamiltonian.

Next, we focus on the very interesting $(n, p)$ side of the IVSM excitations, which is the source of speculations in [4] concerning the importance of the GTGR region and beyond, that is, excitation energies in the range $10 \leqslant E \leqslant 30 \mathrm{MeV}$, for the two-neutrino double beta decay of ${ }^{116} \mathrm{Cd}$. The results of the realistic calculations are shown in Fig. 3, where,

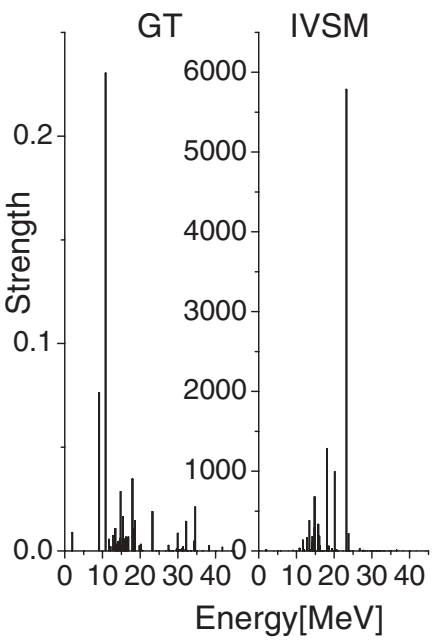

FIG. 3. Results of the realistic calculation for the $\mathrm{GT}^{+}$and $\mathrm{IVSM}^{+}$strengths corresponding to the ${ }^{116} \mathrm{Sn}(n, p){ }^{116} \mathrm{In}$ chargeexchange reaction. The horizontal axes and the units are the same as those of Fig. 1. IVSM transitions have been calculated by excluding $\Delta N=0$ contributions.

again for the sake of the comparison we have removed the contributions with $\Delta N=0$ from the intensity of the IVSM mode. There, the $\mathrm{IVSM}^{+}$strength is confined in the energy range $E \leqslant 30 \mathrm{MeV}$. Notice that, for this branch of decay, seen through the $(n, p)$ charge-exchange reaction on ${ }^{116} \mathrm{Sn}$, the IVSM resonance appears as a narrow state at energy $E \approx 23$ $\mathrm{MeV}$, in very good agreement with the estimate made in [4]. This resonance is also present in the results of the schematic calculations roughly at the same energy.

It is interesting to notice that in [4] the equivalent $\mathrm{GT}^{+}$ strength measured at energies below $30 \mathrm{MeV}$ was some 11 units. It was speculated that six units of this would be genuine $\mathrm{GT}^{+}$strength and the rest, five units, would come from the $\mathrm{IVSM}^{+}$mode. The six units of $\mathrm{GT}^{+}$strength would then connect strongly to the $\mathrm{GT}^{-}$strength at energies in the GTGR

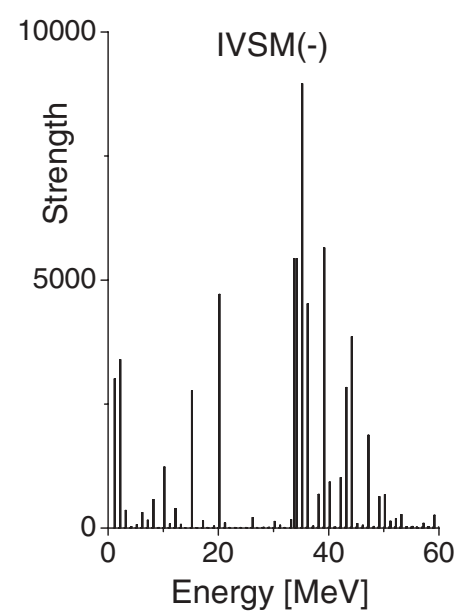

FIG. 4. Results of the realistic calculation for the IVSM $^{-}$ strength. IVSM transitions have been calculated by including all possible $\Delta N=0,2$ contributions. 


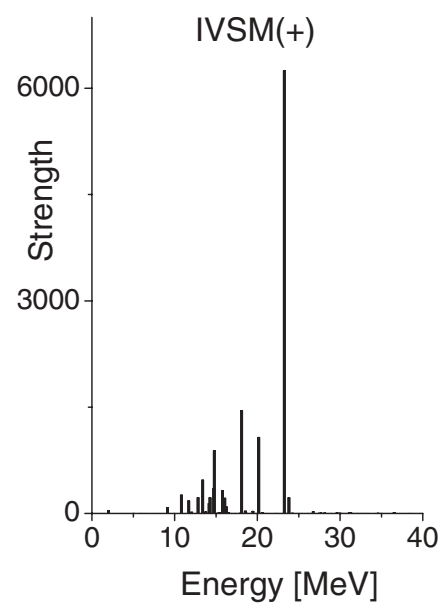

FIG. 5. Results of the realistic calculation for the IVSM $^{+}$ strength. IVSM transitions have been calculated by including all possible $\Delta N=0,2$ contributions.

region and beyond and thus affect substantially the double beta decay rate in ${ }^{116} \mathrm{Cd}$.

Finally, and in order to estimate the total strength distribution for IVSM modes at low energies, we show in Figs. 4 and 5 the intensity of IVSM $^{ \pm}$transitions, including $\Delta N=0$ configurations. The calculated total strengths, including all transitions, are of the order of $6.936 \times 10^{4} \mathrm{fm}^{4}\left(\mathrm{IVSM}^{-}\right.$ mode) and $1.275 \times 10^{4} \mathrm{fm}^{4}$ (IVSM ${ }^{+}$mode), respectively. As seen from Figs. 4 and 5, the inclusion of $\Delta N=0$ transitions does not affect significantly the total strength of the IVSM $^{+}$ mode, and the observation is valid for the strength distribution. However, a more drastic change is observed, particularly, in the low-energy part of the strength distribution belonging to the IVSM $^{-}$mode. For it, also the total strength is drastically affected.

According to our present analysis it would seem that at the GTGR energy and beyond a substantial amount of strength would come from the IVSM $^{+}$and practically none would come from the $\mathrm{GT}^{+}$branch. Hence the calculations produce a negligible amount of $\mathrm{GT}^{+}$strength in the region around the GTGR, thus excluding any sizable effect on the double beta decay rate of ${ }^{116} \mathrm{Cd}$ from energies at and beyond the GTGR energy.

Finally, some words about the overall quality of the calculations. In the realistic Hamiltonian the tensor terms do act upon the GT and IVSM modes, since we are using the Bonn potential to generate the two-body interactions. In the calculations of [12] a larger amount of IVSM strength was found at very low energies. It could very well be that the tensor component of the Bonn interaction is weaker than the one used in [12], which was introduced phenomenologically. Additional renormalization of this interaction may enhance the effects of the IVSM mode at extremely low energies.

\section{SUMMARY AND CONCLUSIONS}

In this work we have studied the possible admixture between the GT and IVSM modes in ${ }^{116} \mathrm{In}$. The question which we have addressed may be of relevance for the experimental search of GT strength at energies higher than the energy of the GTGR. We have developed our analysis starting from a purely schematic situation consisting of an extreme single-particle model space. The results obtained in this extreme model space show that the admixture between GT and IVSM modes is suppressed by the large energy difference between both modes. In order to verify this suppression, we have extended the calculations to a more realistic but still schematic situation by performing a full pnQRPA calculation in a nondegenerate single-particle model space, using a separable interaction and including pairing. We found the same sort of suppression in this case, implying that the distribution of intensity for each mode may indeed be separated from the other. As a final step we have performed realistic calculations by invoking a pnQRPA treatment of the $1^{+}$states in ${ }^{116} \mathrm{In}$, in a Woods-Saxon basis and with realistic interactions constructed with the Bonn-A potential. The realistic calculations were performed for both the $(p, n)$ and $(n, p)$ mechanisms, leading to $1^{+}$states in ${ }^{116} \mathrm{In}$ from ${ }^{116} \mathrm{Sn}$ and ${ }^{116} \mathrm{Cd}$, respectively. As a preliminary test, we have compared the results of the schematic and realistic calculations for the dominant $(p, n)$ branch of the excitations and found that they are consistent.

We may conclude our analysis by pointing out that: (i) the mixing between the GT and IVSM modes does not appear in the models which we have considered (in this respect our results seem to agree with the analysis of [11]) and (ii) the strength found in the $(n, p)$ branch of the excitations, at energies at and above the energy of the GTGR [4], may not be due to the admixture of the $\mathrm{GT}^{+}$and $\mathrm{IVSM}^{+}$modes, but rather it may solely be due to the excitation of the $\mathrm{IVSM}^{+}$mode.

However, one should take this conclusion with caution, since in the analysis of [4] the GT and IVSM strengths are not extracted separately.

Hence, in our calculations we do not find any substantial contribution to the two-neutrino double beta decay of ${ }^{116} \mathrm{Cd}$ at and beyond the GTGR region of energies.

\section{ACKNOWLEDGMENTS}

This work has been supported by the National Research Council (Consejo Nacional de Investigaciones Científicas y Técnicas) (PIP 0740) and the Agencia Nacional de Promoción Científica y Tecnológica of Argentina and by the Academy of Finland under the Finnish Center of Excellence Program 2006-2011 (Nuclear and Accelerator Based Program at the University of Jyväskylä). O.C. thanks with pleasure the hospitality of the Department of Physics of the University of Jyväskylä, Finland.
[1] S. Rakers et al., Phys. Rev. C 70, 054302 (2004); E.-W. Grewe et al., ibid. 76, 054307 (2007); S. Rakers et al., ibid. 71, 054313 (2005); E.-W. Grewe et al., ibid. 77, 064303 (2008); 78, 044301 (2008); H. Dohmann et al., ibid. 78, 041602 (2008).
[2] D. Frekers, AIP CP 1180, 40 (2009).

[3] M. Sasano et al., Phys. Rev. C 79, 024602 (2009).

[4] M. Sasano et al., Phys. Rev. C 85, 061301(R) (2012); AIP CP 1180, 40 (2009). 
[5] J. Suhonen and O. Civitarese, Phys. Rep. 300, 123 (1998).

[6] J. D. Vergados, Phys. Rep. 361, 1 (2002).

[7] H. Ejiri, Phys. Rep. 338, 265 (2000).

[8] M. Doi and T. Kotani, Prog. Theor. Phys. 87, 1207 (1992).

[9] F. T. Avignone III, S. R. Elliott, and J. Engel, Rev. Mod. Phys. 80, 481 (2008).

[10] V. Tretyak and Yu. G. Zdesenko, At. Data Nucl. Data Tables 80, 83 (2002).

[11] I. Hamamoto and H. Sagawa, Phys. Rev. C 62, 024319 (2000).
[12] C. L. Bai, H. Q. Zhang, X. Z. Zhang, F. R. Xu, H. Sagawa, and G. Colo, Phys. Rev. C 79, 041301(R) (2009).

[13] T. Wakasa et al., Phys. Rev. C 55, 2909 (1997).

[14] K. Yako et al., Phys. Lett. B 615, 193 (2005).

[15] K. Holinde, Phys. Rep. 68, 121 (1981).

[16] Aa. Bohr and B. R. Mottelson, Nuclear Structure (W. A. Benjamin, New York, 1972), Vol. 1.

[17] O. Civitarese, R. J. Liotta, and M. E. Mosquera, Phys. Rev. C 78, 064308 (2008). 